\title{
Hepatic arterial phase and portal venous phase computed tomography for dose calculation of stereotactic body radiation therapy plans in liver cancer: a dosimetric comparison study
}

Jianghong Xiao ${ }^{1,2+}$, Yan $\mathrm{Li}^{2 \dagger}$, Qingfeng Jiang ${ }^{1,2+}$, Lan Sun ${ }^{2 \dagger}$, Fraser Henderson $\mathrm{Jr}^{3}$, Yongsheng Wang ${ }^{2}$, Xiaogin Jiang ${ }^{1,2}$, Guangjun $\mathrm{Li}^{1,2}$ and Nianyong Chen ${ }^{1,2^{*}}$

\begin{abstract}
Purpose: To investigate the effect of computed tomography (CT) using hepatic arterial phase (HAP) and portal venous phase (PVP) contrast on dose calculation of stereotactic body radiation therapy (SBRT) for liver cancer.

Methods: Twenty-one patients with liver cancer were studied. HAP, PVP and non-enhanced CTs were performed on subjects scanned in identical positions under active breathing control (ABC). SBRT plans were generated using seven-field three-dimensional conformal radiotherapy (7 F-3D-CRT), seven-field intensity-modulated radiotherapy (7 F-IMRT) and single-arc volumetric modulated arc therapy (VMAT) based on the PVP CT. Plans were copied to the HAP and non-enhanced CTs. Radiation doses calculated from the three phases of CTs were compared with respect to the planning target volume (PTV) and the organs at risk (OAR) using the Friedman test and the Wilcoxon signed ranks test.
\end{abstract}

Results: SBRT plans calculated from either PVP or HAP CT, including 3D-CRT, IMRT and VMAT plans, demonstrated significantly lower $(p<0.05)$ minimum absorbed doses covering $98 \%, 95 \%, 50 \%$ and $2 \%$ of PTV (D98\%, D95\%, D50\% and D2\%) than those calculated from non-enhanced CT. The mean differences between PVP or HAP CT and non-enhanced CT were less than $2 \%$ and $1 \%$ respectively. All mean dose differences between the three phases of CTs for OARs were less than $2 \%$.

Conclusions: Our data indicate that though the differences in dose calculation between contrast phases are not clinically relevant, dose underestimation (IE, delivery of higher-than-intended doses) resulting from CT using PVP contrast is larger than that resulting from CT using HAP contrast when compared against doses based upon non-contrast CT in SBRT treatment of liver cancer using VMAT, IMRT or 3D-CRT.

Keywords: Liver cancer, Contrast agent, Dose calculation, Hepatic arterial phase CT, Portal venous phase CT, Stereotactic body radiation therapy, Volumetric modulated arc therapy

\footnotetext{
* Correspondence: nychenyy@gmail.com

${ }^{\dagger}$ Equal contributors

'Department of Radiation Oncology, Cancer Center, West China Hospital,

Sichuan University, Chengdu, Sichuan 610041, PR China

${ }^{2}$ Center for Radiation Physics and Technology, Cancer Center, West China

Hospital, Sichuan University, Chengdu, Sichuan 610041, PR China

Full list of author information is available at the end of the article
} 


\section{Introduction}

Hepatocellular carcinoma (HCC) is the fifth most common cancer worldwide [1]. Moreover, liver is a common site for metastases from a variety of primary malignancies [2]. Generally, surgery is acknowledged to be the most effective treatment for liver cancer, though only $20-40 \%$ of HCC patients may benefit from radical therapies [3]. By time of diagnosis only 10-20\% of metastatic liver cancer cases undergo surgical resection [4]. However, stereotactic body radiation therapy (SBRT), which includes the application of volumetric modulated arc therapy (VMAT), has been an important addition to the arsenal for treatment of liver cancer [5-7].

SBRT is a technique designed to deliver radiation precisely to delineated tumor areas. However, tactics used to define a gross tumor volume (GTV) might vary significantly among professionals, centers and levels of experiences [8]. Use of contrast in hepatic arterial phase (HAP) and portal venous phase (PVP) computed tomography $(\mathrm{CT})$ has improved detection accuracy in liver cancer $[9,10]$. However, contrasted imaging may adversely influence the prescribed SBRT dose distribution. To quantify the influence of contrast on dose calculation, two modes of investigation have been used. One is based on phantoms, or mathematical algorithms, designed to take into account parameters that include photon beam energies, molarities and contrast agent expansion [11,12]. Human studies are another mode, and they have revealed a negligible effect on dose calculation in regions with low contrast agent penetration [13-15]. However, it was reported that in treatment of upper abdominal tumors contrast agent is responsible for greater than $2 \%$ increase in monitor units (MUs) of three-dimensional conformal radiotherapy (3D-CRT) [16], but that the effect of contrast agent on dose calculation decreased with an increasing number of incident beams [12]. Since VMAT plans are designed using a larger number of segments (typically, there are 91 segments in a single-arc VMAT plan), the effect of contrast agent on VMAT plans is unknown.

Because liver has a dual blood supply from the hepatic artery and portal vein, distribution of contrast agent should differ between HAP and PVP CTs. It would then be reasonable to suspect that radiotherapy plans, based on either HAP or PVP CT, may differ as well and that dosing errors may result. To our knowledge, there are no studies on the effect of different CT phases for dose calculation of SBRT treatment plans, especially using VMAT, in liver cancer. We therefore conducted this study to query the influence of HAP and PVP CT on dose calculation of SBRT plans in liver cancer.

\section{Material and methods \\ Eligibility}

Twenty-one liver cancer patients were enrolled from a single institution. Included patients had no more than three lesions, and liver lesions were no larger than $6 \mathrm{~cm}$ in diameter. All patients were ruled-out for surgical resection before being enrolled (Table 1).

\section{Acquisition of CT}

Each patient was immobilized in a stereotactic body frame (SBF, Elekta Oncology System, Sweden) in the supine position with arms raised above the head. Patients were trained to adapt to active breathing control (ABC) beforehand at moderate deep inspiration breath-hold with $75 \%$ of maximum inspiratory volume. Non-enhanced CT covering the total liver volume was obtained (Philips Gemini GXL, $120 \mathrm{kVp}, 90 \mathrm{mAs}$ ) at $3 \mathrm{~mm}$ slice thickness under ABC. 25-30 seconds after intravenous bolus injection of $120 \mathrm{ml}$ of the contrast agent (Iopamiro370, a non-ionic Xray contrast agent; main ingredient: iopamidol $370 \mathrm{mgI} / \mathrm{ml}$, Bracco S.P.A., Italy) with a power injector at a rate of 4-5 $\mathrm{ml} /$ second, the HAP CT was scanned; PVP CT was obtained 55-60 seconds after the injection.

\section{Region of interest contouring}

After the image acquisition, CTs obtained in three phases were transferred to a radiotherapy planning system (Pinnacle3 9.0, Philips Inc., USA). Representative imaging demonstrating the three phases is provided in Figure 1.

The GTV and organs at risk (OAR) were delineated on the PVP CT. GTV was expanded by a $5-\mathrm{mm}$ radial and a $10-\mathrm{mm}$ cranial-caudal margin to create the

Table 1 Basic and clinical characteristics of selected patients in present study $(\mathbf{n}=\mathbf{2 1})$

\begin{tabular}{ll}
\hline Age & \\
\hline Median & 48 \\
Range & $32-77$ \\
Gender & \\
Male & $15(71.4 \%)$ \\
Female & $6(29.6 \%)$ \\
Pathology & \\
Primary hepatocellular carcinoma & $9(42.9 \%)$ \\
Metastatic liver cancer & $12(57.1 \%)$ \\
PTV Volume $\left(\mathrm{cm}^{3}\right)$ & \\
$0-<50$ & $14(66.7 \%)$ \\
$50-<100$ & $4(19.0 \%)$ \\
$100-<150$ & $2(9.5 \%)$ \\
$150-200$ & $1(4.8 \%)$ \\
\hline
\end{tabular}




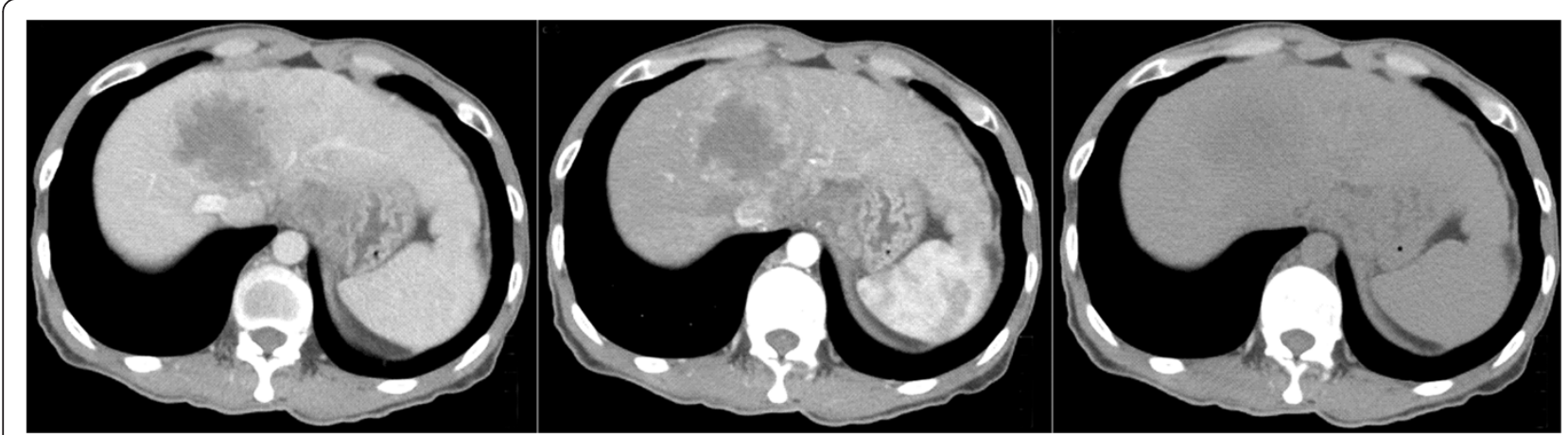

Figure 1 Three phases of CTs for the same patient. Left: portal venous phase (PVP) CT; Middle: hepatic arterial phase (HAP) CT; Right: non-enhanced $C T$.

planning target volume (PTV). Liver, spinal cord, stomach, bowel and kidneys were contoured as the OARs.

\section{Treatment planning}

SBRT plans were designed on the PVP CT using 3DCRT, IMRT and VMAT respectively for each patient. All plans were executed using a $6 \mathrm{MV}$ photon beam from a linear accelerator (Synergy, Elekta, Sweden). The prescription doses were 48 Gy in 4 fractions.

3D-CRT plans were generated using seven coplanar static-fields. According to the guidance from RTOG 0236 recommendations for lung SBRT, no additional margin at the edges of the blocks or multi-leaf collimator (MLC) jaws beyond the PTV was considered. In this study, the minimum absorbed dose covering 95\% of PTV (D95\%) met or exceeded $48 \mathrm{~Gy}$; total patient tissue volume (not including PTV) receiving $\geq 110 \%$ of the prescription dose could not exceed $1.0 \mathrm{~cm}^{3}$; liver mean dose (LMD) was less than $22 \mathrm{~Gy}$; the percentage of right kidney volume receiving $\geq 15$ Gy (V15) was less than 33\%; dose to spinal cord could not exceed $18 \mathrm{~Gy}$; maximum volume of bowel and stomach receiving $\geq 30 \mathrm{~Gy}$ (V30) less than $0.5 \mathrm{~cm}^{3}$.

IMRT plans (7 F-IMRT) were generated using the same fields as the 3D-CRT plans, optimized using Direct Machine Parameter Optimization (DMPO). Additional parameters were as follows: the minimum segment area was $4 \mathrm{~cm}^{2}$; the minimum segment MU was 5; the maximum number of segments was 50 ; total patient tissue volume receiving $\geq 110 \%$ of the prescription dose could not exceed $1.0 \mathrm{~cm}^{3}$. Remaining parameters were the same as in 3D-CRT plans.

VMAT plans were generated using a single arc $\left(181^{\circ}\right.$ $\left.180^{\circ}\right)$. Other parameters were identical to those of the IMRT plan.

All doses were calculated using a collapsed-cone convolution (CC) algorithm with grid size $0.4 \times 0.4 \times$ $0.4 \mathrm{~cm}^{3}$, and all doses were calculated using the electron densities converted from CT number (HU) based on the Model 062 M Electron density phantom composed of eight different tissue equivalent inserts (doses to tissue). After the completion of plans using PVP CT, prescription doses were normalized to MUs. All contours and plans were copied to the HAP and non-enhanced CTs. Thereafter, the radiation doses were recalculated.

To verify dose differences caused by contrast agent or other factors (such as algorithm, inconsistency of patients' positions, etc.), the density of three phases of CTs (PVP, HAP and non-enhanced) were specified as the density of water. Then the doses were recalculated (doses to water).

\section{Statistical analysis}

To evaluate the equivalence of the patients' positions and coordinates among these CTs after image fusion, $\mathrm{Y}$ coordinates (cranial-caudal axis) of the diaphragmatic dome and the source to surface distances (SSD) were recorded and analyzed. Dose parameters in the targets and OARs among these CTs were compared, including the D98\%, $\mathrm{D} 95 \%, \mathrm{D} 50 \%$, D2\%, heterogeneity index $(\mathrm{HI}=(\mathrm{D} 2 \%$ D98\%)/D50\%) and conformity index (CI) where CI is given by the following equation: $\mathrm{CI}=\left(\mathrm{TV}_{95 \%} \times \mathrm{TV}_{95 \%}\right) /$ $\left.\left(\mathrm{TV} \times \mathrm{V}_{95 \%}\right)\right)\left[\mathrm{N} . \mathrm{B} . \mathrm{TV}_{95 \%}=\mathrm{PTV}\right.$ volume covered by the 95\% isodose, $\mathrm{TV}=\mathrm{PTV}$, and $\mathrm{V}_{95 \%}=$ volume of the $95 \%$ isodose]. OAR parameters included the $\mathrm{D} 2 \%$ of the right kidney, bowel, stomach and spinal cord; the mean dose of the liver and right kidney; and the percentage of total liver volume receiving $\geq 21$ Gy (V21). GTV was excluded from liver volume. Differences were analyzed by Friedman test or Wilcoxon signed ranks test (SPSS, Release 17.0). $p<$ 0.05 (2-tailed) indicated statistical significance.

\section{Results}

SSD

Differences between SSDs ranged from -8.7 to $8.50 \mathrm{~mm}$ in each beam. Average SSD difference between PVP CT and non-enhanced $\mathrm{CT}$ was $0.23 \pm 1.77 \mathrm{~mm}$ and for HAP CT versus non-enhanced CT was $0.28 \pm 1.77 \mathrm{~mm}$. Diaphragmatic dome coordinates differed with a range of -9.0 to $6.0 \mathrm{~mm}$. Average difference of the diaphragmatic 
dome coordinate was $0.86 \pm 4.15 \mathrm{~mm}$ for PVP CT vs. non-enhanced CT, and $-0.14 \pm 4.60 \mathrm{~mm}$ for HAP CT vs. non-enhanced CT. There were no significant differences among the SSDs or diaphragmatic dome coordinate of the PVP, HAP and non-enhanced CTs $(p>0.05)$.

Hounsfield units (HU) representing the GTV and liver varied significantly $(p<0.05)$ among the PVP, HAP, and non-enhanced CTs. HU values for GTV and liver by imaging phase used, in terms of magnitude, were PVP $\mathrm{CT}>\mathrm{HAP} \mathrm{CT}>$ non-enhanced CT. The mean differences of HUs in GTV and liver among three sets of CTs are as follows: $41.06 \pm 24.74 \mathrm{HU}$ and $58.05 \pm 17.83 \mathrm{HU}$ for PVP CT vs. non-enhanced CT, $22.58 \pm 17.62 \mathrm{HU}$ and $20.23 \pm$ $22.56 \mathrm{HU}$ for the set of HAP CT vs. non-enhanced CT, and $18.48 \pm 14.65 \mathrm{HU}$ and $37.82 \pm 14.82 \mathrm{HU}$ for the phase of PVP CT vs. HAP CT.

\section{PTV}

We calculated significant variation $(p<0.05)$ in $\mathrm{D} 98 \%$, D95\%, D50\% and D2\% among PVP-, HAP- and nonenhanced CT-based plans, regardless of whether 3DCRT, IMRT or VMAT was used. D98\%, D95\%, D50\% and D2\% calculated from the three phases of CTs, by order of magnitude, were as follows: PVP CT $<$ HAP $\mathrm{CT}<$ non-enhanced CT $(p<0.05)$. Maximum differences in D98\%, D95\%, D50\% and D2\% calculated from the plans with three phases of CTs were, respectively, 2.75\%, $2.66 \%, 2.94 \%, 4.00 \%$ (PVP CT vs. non-enhanced CT); $2.10 \%, 2.15 \%, 2.43 \%, 2.72 \%$ (HAP CT vs. non-enhanced CT); and $1.93 \%, 1.86 \%, 1.90 \%, 2.99 \%$ (PVP CT vs. HAP $\mathrm{CT}$ ). Mean differences (MD to tissue), however, were less at 2\% (PVP CT vs. non-enhanced CT), 1\% (HAP CT vs. non-enhanced CT), and 1\% (PVP CT vs. HAP CT) (see Table 2).

\section{OARs}

Analysis of doses to OAR shows LMD calculated from either PVP or HAP CT was significantly lower than those from the non-enhanced CT $(p<0.05)$. However, all mean differences across the three phases of CT in dose to OARs was less than $2 \%$ (Table 3).

With the density specified as the density of water, the mean differences in D98\%, D95\%, D50\% and D2\% for PTV (MD to water) among the three phases of CTs were less than $0.1 \%$; the ratio of MD to water and MD to tissue were less than $6.64 \%$.

Dose differences attributable to the contrast agent were similar among the three kinds of plans (single arc VMAT, 7 F-IMRT and 7 F-3D-CRT, $p>0.05$ ) according to the Friedman test.

\section{Discussion}

The liver possesses a dual blood supply from the hepatic portal vein and hepatic arteries. The hepatic portal vein supplies approximately $75 \%$ of the liver's blood flow, and the hepatic artery accounts for the remainder. Moreover, the liver is the dominant organ for congregation of contrast agent. After intravenous bolus injection, contrast agent transported via hepatic artery arrives 20-30 seconds earlier to the liver than if transported via portal vein, and this differential explains the difference seen between HAP and PVP imaging. Studies have confirmed that utilizing both PVP and HAP CTs elevates the rates of detection $[9,10]$. Therefore, the PVP and HAP CTs are often applied during the simulation stage of liver cancer radiotherapy treatment. Hounsfield units represent a tissue's electron density via $x$-ray attenuation, and as such, are a critical and contributory component of dose calculation in the planning system. In this study, we found that the HUs of the GTV and liver in both PVP and HAP CTs were higher than those in the nonenhanced CT; moreover, the increase of HUs in the PVP CT was more obvious than that in other phases.

In this study, SSDs varied in patients similarly to our previous report on lung cancer [13], while there was more variation than what has been seen on head and neck cancer [14]. Due to the significant cranial-caudal displacement of liver resulting from respiratory motion [17], we assessed the Y-axes coordinates of the diaphragmatic dome. Both the mean value and range of the diaphragmatic dome's displacement were much smaller with $A B C$ than without it. There were no significant differences in either SSDs or displacement of the diaphragmatic dome among these phases of CTs. Therefore, patients were considered to have been scanned in nearly identical positions; the influence of positioning errors among these CTs was negligible.

Shibamoto et al. reported an increase of $51 \pm 17 \mathrm{HU}$ of enhanced liver CT over non-enhanced. Mean increases of MUs were more than $2 \%$, and the maximum increase was 7.6 MU for upper abdominal tumors [16]. In contrast to their results, our data showed that in the SBRT plans, the dose differences to PTV between the PVP and non-enhanced CTs were lower than 2\%; however, the dose differences to PTV between the HAP and nonenhanced CTs were lower than $1 \%$. We speculate that $\mathrm{ABC}$ technique explains the divergence. $\mathrm{ABC}$ works to coordinate scanning with cycles in respiratory movement, and thus we feel that our analysis of the treatment effect of contrast agent in imaging is better assessed employing the $\mathrm{ABC}$ technology. Choi et al. found the GTV HUs of head and neck increased by an average of 55 HUs, and that PTV doses differed less than 1\% (enhanced CT vs. non-enhanced CT) [14]. These dose differences are similar to the dose changes between the HAP and non-enhanced CTs; however, they are less than the dose differences between the PVP and non-enhanced CTs in our study. Here we speculate that this change in dose 
Table 2 Comparison of PTV doses calculated from PVP, HAP and non-enhanced CTs

\begin{tabular}{|c|c|c|c|c|c|c|c|c|c|c|}
\hline & $\mathrm{Dp}$ & $\mathrm{Da}$ & Dn & $P(p, a, n)^{*}$ & (Dn-Dp)/Dn(\%) & $P(p, n) \#$ & (Dn-Da)/Dn(\%) & $P(a, n) \#$ & (Da-Dp)/Dn(\%) & $P(p, a) \#$ \\
\hline \multicolumn{11}{|l|}{ 3D-CRT } \\
\hline $\mathrm{Cl}$ & $0.84 \pm 0.07$ & $0.83 \pm 0.07$ & $0.83 \pm 0.07$ & 0.116 & $-1.15 \pm 2.44$ & - & $-0.49 \pm 1.72$ & - & $-0.66 \pm 1.00$ & - \\
\hline $\mathrm{HI}$ & $0.17 \pm 0.02$ & $0.17 \pm 0.02$ & $0.17 \pm 0.02$ & 0.117 & $1.04 \pm 4.84$ & - & $-0.50 \pm 5.77$ & - & $1.54 \pm 4.29$ & - \\
\hline D98\% (Gy) & $46.83 \pm 0.28$ & $47.09 \pm 0.41$ & $47.36 \pm 0.46$ & 0.000 & $1.12 \pm 0.78$ & 0.000 & $0.56 \pm 0.51$ & 0.000 & $0.56 \pm 0.58$ & 0.000 \\
\hline D95\% (Gy) & $48.09 \pm 0.06$ & $48.36 \pm 0.29$ & $48.62 \pm 0.40$ & 0.000 & $1.10 \pm 0.76$ & 0.000 & $0.54 \pm 0.58$ & 0.000 & $0.56 \pm 0.56$ & 0.000 \\
\hline D50\% (Gy) & $53.17 \pm 0.52$ & $53.52 \pm 0.57$ & $53.83 \pm 0.75$ & 0.000 & $1.23 \pm 0.81$ & 0.000 & $0.58 \pm 0.54$ & 0.000 & $0.64 \pm 0.56$ & 0.000 \\
\hline D2\% (Gy) & $55.63 \pm 1.14$ & $56.09 \pm 1.28$ & $56.39 \pm 1.43$ & 0.000 & $1.33 \pm 1.21$ & 0.000 & $0.53 \pm 1.09$ & 0.000 & $0.81 \pm 0.93$ & 0.000 \\
\hline \multicolumn{11}{|l|}{ IMRT } \\
\hline $\mathrm{Cl}$ & $0.85 \pm 0.04$ & $0.82 \pm 0.06$ & $0.79 \pm 0.09$ & 0.000 & $-8.60 \pm 9.78$ & 0.000 & $-4.34 \pm 6.09$ & 0.000 & $-4.25 \pm 5.84$ & 0.000 \\
\hline $\mathrm{HI}$ & $0.05 \pm 0.01$ & $0.06 \pm 0.01$ & $0.06 \pm 0.01$ & 0.040 & $4.82 \pm 8.30$ & 0.006 & $2.58 \pm 5.74$ & 0.070 & $2.24 \pm 5.70$ & 0.111 \\
\hline D98\% (Gy) & $47.60 \pm 0.20$ & $47.85 \pm 0.30$ & $48.06 \pm 0.45$ & 0.000 & $0.94 \pm 0.68$ & 0.000 & $0.43 \pm 0.49$ & 0.000 & $0.51 \pm 0.43$ & 0.000 \\
\hline D95\% (Gy) & $48.05 \pm 0.08$ & $48.31 \pm 0.23$ & $48.54 \pm 0.35$ & 0.000 & $1.01 \pm 0.72$ & 0.000 & $0.46 \pm 0.50$ & 0.000 & $0.55 \pm 0.51$ & 0.000 \\
\hline D50\% (Gy) & $49.24 \pm 0.30$ & $49.54 \pm 0.43$ & $49.80 \pm 0.48$ & 0.000 & $1.11 \pm 0.83$ & 0.000 & $0.52 \pm 0.62$ & 0.000 & $0.59 \pm 0.53$ & 0.000 \\
\hline D2\% (Gy) & $50.28 \pm 0.45$ & $50.61 \pm 0.51$ & $50.91 \pm 0.60$ & 0.000 & $1.23 \pm 0.99$ & 0.000 & $0.59 \pm 0.74$ & 0.000 & $0.64 \pm 0.66$ & 0.000 \\
\hline \multicolumn{11}{|l|}{ VMAT } \\
\hline $\mathrm{Cl}$ & $0.81 \pm 0.08$ & $0.79 \pm 0.08$ & $0.78 \pm 0.10$ & 0.000 & $-4.93 \pm 6.74$ & 0.000 & $-2.38 \pm 4.89$ & 0.002 & $-2.54 \pm 2.91$ & 0.000 \\
\hline $\mathrm{HI}$ & $0.07 \pm 0.02$ & $0.08 \pm 0.02$ & $0.08 \pm 0.02$ & 0.137 & $1.92 \pm 6.65$ & - & $-0.51 \pm 4.73$ & - & $2.43 \pm 4.11$ & - \\
\hline D98\% (Gy) & $47.45 \pm 0.23$ & $47.67 \pm 0.32$ & $47.89 \pm 0.47$ & 0.000 & $0.90 \pm 0.64$ & 0.000 & $0.45 \pm 0.45$ & 0.000 & $0.45 \pm 0.38$ & 0.000 \\
\hline D95\% (Gy) & $48.06 \pm 0.04$ & $48.28 \pm 0.21$ & $48.51 \pm 0.31$ & 0.000 & $0.93 \pm 0.66$ & 0.000 & $0.47 \pm 0.42$ & 0.000 & $0.46 \pm 0.44$ & 0.000 \\
\hline D50\% (Gy) & $49.98 \pm 0.41$ & $50.27 \pm 0.51$ & $50.52 \pm 0.51$ & 0.000 & $1.06 \pm 0.79$ & 0.000 & $0.49 \pm 0.51$ & 0.000 & $0.58 \pm 0.52$ & 0.000 \\
\hline D2\% (Gy) & $51.19 \pm 0.62$ & $51.54 \pm 0.76$ & $51.78 \pm 0.80$ & 0.000 & $1.13 \pm 0.87$ & 0.000 & $0.46 \pm 0.53$ & 0.000 & $0.68 \pm 0.59$ & 0.000 \\
\hline
\end{tabular}

Abbrevations: PVP, portal venous phase; HAP, hepatic arterial phase. Dp, Da and Dn were the dose calculated from portal venous phase, hepatic arterial phase and non-enhanced phase CTs respectively. $98 \%, 95 \%, 50 \%$ and $2 \%$, were the minimum absorbed dose that covers $98 \%, 95 \%, 50 \%$ and $2 \%$ of the volume of the PTV respectively. $\mathrm{Cl}$, the conformity index; $\mathrm{HI}$, the heterogeneity index. ${ }^{*}$, Friedman test; \#, Wilcoxon signed ranks test.

underestimation may be caused by contrast agent distribution varied in different phases. Moreover HU values by order of magnitude for GTV and liver were PVP CT > HAP CT $>$ non-enhanced CT $(p<0.05)$. Therefore, the resulting data encourages us to further clarify the effect of contrast agent application on the dose underestimation for the plans of radiotherapy.

Ramm et al. had found in a phantom-based study that the effect of contrast agent decreased with an increase of the number of incident beams [12]. In Ramm et al's study, the plans were created with a single photon beam or two opposing photon beams or an isocentric four-field box technique. The number of incident beams was less than that in our study (VMAT 91 segments; IMRT $\leq 50 \mathrm{seg}$ ments; 3D-CRT 7 beams). Although the VMAT plans had the most segments or beams, compared with nonenhanced CT, the dose underestimations attributable to the contrast agent showed no significant difference across the three plan types $(p>0.05)$. It is possible that when the number of beams or segments increases to a certain threshold value, the dose effects caused by the contrast agent will tend to be consistent. This question deserves further investigation.
There was concern that the accuracy of the $\mathrm{CC}$ algorithm might affect the accuracy of dose underestimation in our study. Therefore calibrated ionization chamber and radiochromic EBT type films in a homogenous polystyrene phantom and in heterogeneous lung phantoms were used for evaluating the accuracy of the algorithm [18]. The result indicated some small difference between the $\mathrm{CC}$ and Monte Carlo (MC) algorithms in the measurements. Knöös et al. also reported that when the average dose in the PTV for prostate cases calculated by the $\mathrm{MC}$ was set to $100 \%$, the average dose, D95\%, and D5\% of PTV calculated by the Pinnacle CC algorithm were $100 \%, 98.2 \%$, and $101.4 \%$ respectively [19]. Because prostatic tissue and liver are similarly homogenous we believe that the algorithm is translatable from prostate to liver and that the effect of the algorithm on the dose calculation for SBRT plans using VMAT or IMRT or 3D CRT is negligible. The results in this study indicated that when the density of PVP, HAP, and non-enhanced CTs were specified as the density of water, the MD-to-water among three phases of CTs were less than $0.1 \%$, the ratio of MD-to-water and MD-to-tissue were less than 6.64\%. This finding further confirmed that the dose differences 
Table 3 Comparison of OARs' doses calculated from PVP, HAP and non-enhanced CTs

\begin{tabular}{|c|c|c|c|c|c|c|c|c|c|c|}
\hline & $\mathrm{Dp}$ & $\mathrm{Da}$ & Dn & $P(p, a, n)^{*}$ & $(\mathrm{Dn}-\mathrm{Dp}) / \mathrm{Dn}(\%)$ & $P(p, n) \#$ & $(\mathrm{Dn}-\mathrm{Da}) / \mathrm{Dn}(\%)$ & $P(a, n) \#$ & (Da-Dp)/Dn (\%) & $P(p, a) \#$ \\
\hline \multicolumn{11}{|l|}{ 3D-CRT } \\
\hline Liver V21 (\%) & $12.77 \pm 11.45$ & $12.87 \pm 11.53$ & $12.94 \pm 11.55$ & 0.000 & $1.70 \pm 1.50$ & 0.000 & $0.90 \pm 1.20$ & 0.000 & $0.80 \pm 0.85$ & 0.000 \\
\hline Liver Dmean (Gy) & $8.52 \pm 5.00$ & $8.57 \pm 5.03$ & $8.61 \pm 5.04$ & 0.000 & $1.02 \pm 0.73$ & 0.000 & $0.51 \pm 0.64$ & 0.000 & $0.52 \pm 0.47$ & 0.000 \\
\hline Right kidney D2\%(Gy) & $13.59 \pm 14.36$ & $13.60 \pm 14.35$ & $13.69 \pm 14.48$ & 0.000 & $0.56 \pm 1.29$ & 0.000 & $0.33 \pm 1.10$ & 0.001 & $0.23 \pm 0.58$ & 0.140 \\
\hline Right kidney Dmean (Gy) & $2.87 \pm 3.47$ & $2.87 \pm 3.47$ & $2.89 \pm 3.50$ & 0.201 & $-0.09 \pm 1.01$ & - & $0.18 \pm 0.81$ & - & $-0.27 \pm 0.53$ & - \\
\hline Bowl D2\% (Gy) & $12.83 \pm 8.63$ & $12.84 \pm 8.65$ & $12.86 \pm 8.72$ & 0.040 & $-0.31 \pm 2.50$ & 0.254 & $-0.13 \pm 2.27$ & 0.074 & $-0.18 \pm 1.13$ & 0.268 \\
\hline Stomach D2\% (Gy) & $16.42 \pm 7.62$ & $16.54 \pm 7.66$ & $16.65 \pm 7.69$ & 0.000 & $1.45 \pm 2.33$ & 0.003 & $0.71 \pm 1.40$ & 0.004 & $0.74 \pm 1.31$ & 0.001 \\
\hline Spinal cord D2\% (Gy) & $11.28 \pm 6.79$ & $11.31 \pm 6.79$ & $11.36 \pm 6.81$ & 0.006 & $0.73 \pm 1.38$ & 0.000 & $0.46 \pm 0.88$ & 0.004 & $0.27 \pm 1.06$ & 0.234 \\
\hline \multicolumn{11}{|l|}{ IMRT } \\
\hline Liver V21 (\%) & $25.31 \pm 6.90$ & $25.35 \pm 6.89$ & $25.39 \pm 6.87$ & 0.040 & $0.38 \pm 0.87$ & 0.046 & $0.19 \pm 0.68$ & 0.338 & $0.19 \pm 0.43$ & 0.026 \\
\hline Liver Dmean (Gy) & $10.00 \pm 4.95$ & $10.05 \pm 4.99$ & $10.09 \pm 4.99$ & 0.000 & $0.97 \pm 0.77$ & 0.000 & $0.49 \pm 0.74$ & 0.000 & $0.48 \pm 0.46$ & 0.000 \\
\hline Right kidney D2\% (Gy) & $14.71 \pm 14.88$ & $14.71 \pm 14.89$ & $14.82 \pm 15.02$ & 0.003 & $0.32 \pm 1.22$ & 0.002 & $0.30 \pm 1.21$ & 0.006 & $0.01 \pm 0.50$ & 0.050 \\
\hline Right kidney Dmean (Gy) & $3.04 \pm 3.12$ & $3.03 \pm 3.12$ & $3.06 \pm 3.15$ & 0.090 & $0.02 \pm 1.00$ & - & $0.29 \pm 0.84$ & - & $-0.27 \pm 0.54$ & - \\
\hline Bowl D2\% (Gy) & $10.99 \pm 6.88$ & $11.02 \pm 6.90$ & $11.09 \pm 6.93$ & 0.000 & $0.90 \pm 0.86$ & 0.000 & $0.65 \pm 0.88$ & 0.002 & $0.26 \pm 0.69$ & 0.178 \\
\hline Stomach D2\% (Gy) & $11.73 \pm 6.99$ & $11.80 \pm 7.03$ & $11.85 \pm 7.07$ & 0.000 & $0.89 \pm 1.90$ & 0.012 & $0.39 \pm 1.23$ & 0.008 & $0.50 \pm 1.28$ & 0.002 \\
\hline Spinal cord D2\% (Gy) & $9.09 \pm 4.50$ & $9.12 \pm 4.49$ & $9.17 \pm 4.50$ & 0.003 & $0.99 \pm 1.64$ & 0.003 & $0.57 \pm 1.13$ & 0.001 & $0.42 \pm 0.95$ & 0.195 \\
\hline \multicolumn{11}{|l|}{ VMAT } \\
\hline Liver V21 (\%) & $20.71 \pm 7.27$ & $20.75 \pm 7.27$ & $20.77 \pm 7.26$ & 0.017 & $0.35 \pm 0.71$ & 0.018 & $0.14 \pm 0.47$ & 0.355 & $0.21 \pm 0.34$ & 0.005 \\
\hline Liver Dmean (Gy) & $10.10 \pm 5.24$ & $10.16 \pm 5.28$ & $10.20 \pm 5.29$ & 0.000 & $0.97 \pm 0.67$ & 0.000 & $0.45 \pm 0.59$ & 0.000 & $0.52 \pm 0.43$ & 0.000 \\
\hline Right kidney D2\% (Gy) & $14.30 \pm 14.80$ & $14.33 \pm 14.83$ & $14.41 \pm 14.87$ & 0.000 & $0.68 \pm 1.08$ & 0.000 & $0.38 \pm 0.89$ & 0.000 & $0.31 \pm 0.48$ & 0.000 \\
\hline Right kidney Dmean (Gy) & $3.34 \pm 3.55$ & $3.34 \pm 3.55$ & $3.36 \pm 3.56$ & 0.187 & $-0.03 \pm 1.03$ & - & $0.14 \pm 0.76$ & - & $-0.17 \pm 0.41$ & - \\
\hline Bowl D2\% (Gy) & $11.87 \pm 7.28$ & $11.90 \pm 7.32$ & $11.98 \pm 7.35$ & 0.000 & $0.95 \pm 0.87$ & 0.000 & $0.81 \pm 1.02$ & 0.001 & $0.14 \pm 0.94$ & 0.206 \\
\hline Stomach D2\% (Gy) & $11.53 \pm 8.30$ & $11.65 \pm 8.47$ & $11.65 \pm 8.40$ & 0.000 & $0.78 \pm 1.60$ & 0.001 & $0.08 \pm 1.95$ & 0.031 & $0.70 \pm 1.12$ & 0.000 \\
\hline Spinal cord D2\% (Gy) & $9.92 \pm 3.79$ & $9.93 \pm 3.79$ & $9.95 \pm 3.80$ & 0.013 & $0.24 \pm 1.18$ & 0.010 & $0.21 \pm 0.56$ & 0.031 & $0.04 \pm 0.77$ & 0.081 \\
\hline
\end{tabular}

Abbrevations: V21, the percentage liver volume received more than 21 Gy; Dmean, the mean dose; D2\%, the minimum absorbed dose that covers $2 \%$ of the volume of a certain OAR. The others as in Table 2.

*, Friedman test; \#, Wilcoxon signed ranks test. 
were due to the effect of using contrast agent and not the algorithm.

In conclusion, SBRT plans for treating liver cancer, whether with VMAT or IMRT or 3D-CRT, are susceptible to dose underestimations caused by contrast agent from the PVP CT that are larger than those from the HAP CT when compared with non-enhanced CT. Nevertheless, the detection rate in HAP CT is higher than that in PVP CT [9]. Therefore, it is still recommended HAP CT to be applied for patient simulation in the treatment planning in liver cancer.

\section{Competing interest}

The authors declare that the article content was composed in the absence of any commercial or financial relationships that could be construed as a potential conflict of interest.

\section{Authors' contributions}

JX, YL, QJ and LS contributed equally to this work, participated in the design of the study, carried out the study, performed the statistical analysis, and drafted the manuscript; YW, XJ and GL: helped to carried out the study; FH: reviewed and edited the manuscript; NC: conceived, designed, and coordinated the study, and edited and reviewed the manuscript. All authors read and approved the final manuscript.

\section{Author details}

'Department of Radiation Oncology, Cancer Center, West China Hospital, Sichuan University, Chengdu, Sichuan 610041, PR China. ${ }^{2}$ Center for Radiation Physics and Technology, Cancer Center, West China Hospital, Sichuan University, Chengdu, Sichuan 610041, PR China. ${ }^{3}$ University of Virginia School of Medicine, Charlottesville, Va 22908, USA.

Received: 28 June 2013 Accepted: 6 November 2013 Published: 9 November 2013

\section{References}

1. Llovet JM, Burroughs A, Bruix J: Hepatocellular carcinoma. Lancet 2003, 362:1907-1917.

2. Grover A, Alexander HR Jr: The past decade of experience with isolated hepatic perfusion. Oncologist 2004, 9:653-664.

3. Mazzaferro V, Regalia E, Doci R, Andreola S, Pulvirenti A, Bozzetti F, Montalto F, Ammatuna M, Morabito A, Gennari L: Liver transplantation for the treatment of small hepatocellular carcinomas in patients with cirrhosis. N Engl J Med 1996, 334:693-699.

4. Small R, Lubezky N, Ben-Haim M: Current controversies in the surgical management of colorectal cancer metastases to the liver. Isr Med Assoc J 2007 9:742-747.

5. Rusthoven KE, Kavanagh BD, Cardenes H, Stieber WW, Burri SH, Feigenberg SJ, Chidel MA, Pugh TJ, Franklin W, Kane M, Gaspar LE, Schefter TE: Multiinstitutional phase $\mathrm{I} / \mathrm{ll}$ trial of stereotactic body radiation therapy for liver metastases. J Clin Oncol 2009, 27:1572-1578.

6. Cárdenes HR, Price TR, Perkins SM, Maluccio M, Kwo P, Breen TE, Henderson MA, Schefter TE, Tudor K, Deluca J, Johnstone PA: Phase I feasibility trial of stereotactic body radiation therapy for primary hepatocellular carcinoma. Clin Transl Oncol 2010, 12:218-225.

7. Wang PM, Hsu WC, Chung NN, Chang FL, Fogliata A, Cozzi L: Radiation treatment with volumetric modulated arc therapy of hepatocellular carcinoma patients: early clinical outcome and toxicity profile from a retrospective analysis of 138 patients. Radiat Oncol 2012, 7:207.

8. Giraud P, Elles S, Helfre S, De Rycke Y, Servois V, Carette MF, Alzieu C, Bondiau PY, Dubray B, Touboul E, Housset M, Rosenwald JC, Cosset JM: Conformal radiotherapy for lung cancer: different delineation of the gross tumor volume (GTV) by radiologists and radiation oncologists. Radiother Oncol 2002, 62:27-36.

9. Ichikawa T, Federle MP, Grazioli L, Nalesnik M: Hepatocellular adenoma: multiphasic $\mathrm{CT}$ and histopathologic findings in 25 patients.

Radiology 2000, 214:861-868.
10. Scott DJ, Guthrie JA, Arnold P, Ward J, Atchley J, Wilson D, Robinson PJ: Dual phase helical CT versus portal venous phase $\mathrm{CT}$ for the detection of colorectal liver metastases: correlation with intra-operative sonography, surgical and pathological findings. Clin Radiol 2001, 56:235-242.

11. Robar JL, Ricco SA, Martin MA: Tumour dose enhancement using modified megavoltage photon beams and contrast media. Phys Med Biol 2002, 47:2433-2449.

12. Ramm U, Damrau M, Mose S, Manegold KH, Rahl CG, Böttcher HD: Influence of CT contrast agents on dose calculations in a 3D treatment planning system. Phys Med Biol 2001, 46:2631-2635.

13. Xiao J, Zhang H, Gong Y, Fu Y, Tang B, Wang S, Jiang Q, Li P: Feasibility of using intravenous contrast-enhanced computed tomography (CT) scans in lung cancer treatment planning. Radiother Oncol 2010, 96:73-77.

14. Choi Y, Kim JK, Lee HS, Hur WJ, Hong YS, Park S, Ahn K, Cho H: Influence of intravenous contrast agent on dose calculations of intensity modulated radiation therapy plans for head and neck cancer. Radiother Oncol 2006, 81:158-162

15. Burridge NA, Rowbottom CG, Burt PA: Effect of contrast-enhanced CT scans on heterogeneity corrected dose computations in the lung. J Appl Clin Med Phys 2006, 7:1-12

16. Shibamoto Y, Naruse A, Fukuma $H$, Ayakawa S, Sugie C, Tomita N: Influence of contrast materials on dose calculation in radiotherapy planning using computed tomography for tumors at various anatomical regions: a prospective study. Radiother Oncol 2007, 84:52-55.

17. Case RB, Moseley DJ, Sonke JJ, Eccles CL, Dinniwell RE, Kim J, Bezjak A, Milosevic M, Brock KK, Dawson LA: Interfraction and intrafraction changes in amplitude of breathing motion in stereotactic liver radiotherapy. Int $J$ Radiat Oncol Biol Phys 2010, 77:918-925.

18. Fotina I, Winkler P, Künzler T, Reiterer J, Simmat I, Georg D: Advanced kernel methods vs. Monte Carlo-based dose calculation for high energy photon beams. Radiother Oncol 2009, 93:645-653.

19. Knöös T, Wieslander E, Cozzi L, Brink C, Fogliata A, Albers D, Nyström H, Lassen S: Comparison of dose calculation algorithms for treatment planning in external photon beam therapy for clinical situations. Phys Med Biol 2006, 51:5785-5807.

doi:10.1186/1748-717X-8-264

Cite this article as: Xiao et al:: Hepatic arterial phase and portal venous phase computed tomography for dose calculation of stereotactic body radiation therapy plans in liver cancer: a dosimetric comparison study. Radiation Oncology 2013 8:264.

\section{Submit your next manuscript to BioMed Central and take full advantage of:}

- Convenient online submission

- Thorough peer review

- No space constraints or color figure charges

- Immediate publication on acceptance

- Inclusion in PubMed, CAS, Scopus and Google Scholar

- Research which is freely available for redistribution 